\title{
АКТУАЛЬНАЯ И КЛАССИЧЕСКАЯ ЛИТЕРАТУРА
}

\section{О наследии В. А. Стеклова по классической механике}

\author{
А. В. Борисов, Л. А. Газизуллина, И. С. Мамаев
}

Статья написана для готовящегося к изданию сборника избранных работ Владимира Андреевича Стеклова, озаглавленного составителями «Работы по механике 1902-1909 гг.: Переводы с французского» ${ }^{1}$. Основу сборника, как видно из названия, составили работы В. А. Стеклова по механике (прежде всего - гидродинамике), опубликованные во французских журналах в период 1902-1909 гг. Недоступные ранее на русском языке, эти сочинения до сих пор были не вполне удобны для ознакомления и подробного изучения. Настоящим их переводом и изданием выполняется не совсем обычная задача, в некотором смысле, обратная традиционной, когда фундаментальные работы русских классиков, в свое время не получившие должной известности за рубежом, переводят и популяризуют в западном научном сообществе.

В. А. Стеклов, очевидно, хорошо осознавал и учитывал вопрос доступности своих научных результатов в международных математических кругах: «С половины девяностых годов я стал печатать часть моих работ (большинство на французском языке) в различных заграничных журналах $\langle\ldots\rangle$ и завязал сношения с различными европейскими учеными». ${ }^{2}$ Фактически, на французском языке В.А. Стеклов написал наибольшую часть своих трудов по математике и механике. Почти ничего из этого наследия для русскоязычного читателя переведено не было (в отличие, например, от трудов его учителя, А. М. Ляпунова). Восполнить хоть сколько-нибудь этот пробел представляется особенно важным, памятуя о том, сколь огромный вклад внес в развитие нашей науки В.А.Стеклов, выдающийся научный и общественный деятель, чье имя носит центральный в стране Математический институт РАН.

Получено и принято к печати 17 января 2011 года

Борисов Алексей Владимирович

borisov@rcd.ru

Газизуллина Лариса Александровна

lag@rcd.ru

Мамаев Иван Сергеевич

mamaev@rcd.ru

Институт компьютерных исследований, Удмуртский государственный университет 426034, Россия, г. Ижевск, ул. Университетская, 1

${ }^{1}$ Сост. А.В. Борисов, И. С. Мамаев, А.В.ЦЦыганов, Серия «Библиотека журнала «РХД», М.Ижевск: НИЦ «РХД», Институт компьютерных исследований, 2011.

${ }^{2}$ Стеклов В.А. Воспоминания // Переписка с отечественными математиками. Воспоминания. Серия «Научное наследство», т. XVII. Л.: Наука, 1991. С. 235-299. 
В истории математики имя В.А. Стеклова приобрело всеобщую известность прежде всего благодаря его достижениям в области математической физики. Они связаны с систематическим исследованием краевых задач для дифференциальных уравнений, разработкой специальных методов их решения, проблемами сходимости и разложимости функций в ряды, созданием теории замкнутости и т.д. Этим вопросам посвящены, главным образом, все работы В.А.Стеклова, начиная с 1895 года. В 1901 году он публикует докторскую диссертацию «Общие методы решения основных задач математической физики» [25]. Его книга «Основные задачи математической физики», вышедшая в 1922-1923 гг. (в двух томax), подводит итог этим многолетним исследованиям. ${ }^{3}$ Как известно, некоторые из этих результатов, безусловно прогрессивные в свое время, в дальнейшем были изложены в более общем и законченном виде с помощью новых методов, возникших с развитием современного функционального анализа.

В ряде работ периода 1902-1909 гг. В. А. Стеклов вновь обращается к механике. К сожалению, данные работы известны не столь широко, хотя заслуживают не меньшего внимания. Это статьи [1-8]. В основном они посвящены вопросам из гидродинамики - движению твердого тела с полостями, наполненными жидкостью, теории вихрей, динамике жидкого самогравитирующего эллипсоида и др. Этим исследованиям В.А. Стеклова и посвящена настоящая статья.

Можно сказать, что эти работы составляют отдельную, особо замечательную, главу творчества В. А. Стеклова. Они объединяют в себе накопленный им опыт и кругозор в различных областях математики и механики, написаны под общим их влиянием. С одной стороны, эти труды продолжают и развивают его ранние исследования по гидродинамике, прежде всего магистерскую диссертацию «О движении твердого тела в жидкости» [23]. С другой стороны, в принципах и методах решения задач данные работы уже во многом примыкают к его докторской диссертации, посвященной вопросам математической физики [25]. В работах по механике этого периода В. А. Стеклов существенно расширил тематику своих исследований, их отличает широта постановок, обсуждение прикладного значения полученных результатов.

Примечательно, что эти исследования сохранили до сих пор помимо исторической и определенную научную ценность. Рассмотренные В.А. Стекловым задачи по-прежнему важны в их связи с современными проблемами динамики вихрей и тел в жидкости, динамики и физики планет и другими динамическими приложениями. Некоторые задачи в окончательном и общем виде еще не разрешены и нуждаются в современном пересмоторе. Работы В.А. Стеклова содержат интересный, наводящий на размышления материал. Его оригинальные, содержательные постановки и идеи представляют несомненный интерес для специалистов, создают предпосылки для дальнейших исследований. В этом смысле издание переводов данных сочинений В. А. Стеклова и сейчас можно считать своевременным.

Как уже отмечалось, вопросы гидродинамики, рассмотренные в этих статьях В. А. Стекловым, навеяны научной работой, с которой началась его творческая деятельность, - исследованием уравнений Кирхгофа о движении твердого тела в идеальной несжимаемой жидкости. Результаты этой работы изложены в его магистерской диссертации [23], опуб-

\footnotetext{
${ }^{3}$ Второе издание вышло в 1983 году под редакцией и с предисловием В. С. Владимирова. (См. также очерк: Владимиров В.С., Маркуш И. И. Владимир Андреевич Стеклов - ученый и организатор науки. М.: Наука, Физматлит, 1981. 95 с.)
} 
ликованной в Харькове в 1893 году. Интересно отметить, что уже после печати это сочинение, в общем безусловно важное и интересное, было подвергнуто тщательному разбору А. М. Ляпуновым. Сохранился экземпляр, в который А. М. Ляпунов собственной рукой внес многочисленные исправления, часто указывающие на изрядные неточности и даже ошибки в изложении В.А. Стеклова. С другой стороны, характер этой правки, касающейся и самых мелких, несущественных деталей, указывает на свойственную самому А. М. Ляпунову чрезмерную скрупулезность и сверхтребовательность, из которых проистекает стремление приблизить изложение ученика к собственному идеалу - исключительной строгости и отсутствию малейших пробелов. О недостатках изложения А. М. Ляпунов говорит и в своем отзыве на докторскую диссертацию В. А. Стеклова [23], при этом в заключение он отмечает: «Но все это легко поправимо и не влияет на выводы, делаемые автором». Обсуждения такого рода можно часто встретить в переписке двух ученых. Критические замечания А. М. Ляпунова принимались В. А. Стекловым с глубоким уважением и благодарностью, хотя можно предположить, что ему, человеку совершенно иного темперамента и склада, порой нелегко было учитывать рекомендации А. М. Ляпунова в полной мере.

Во всем этом наглядно прослеживаются особенности взаимоотношений учителя и ученика, видно своеобразие двух выдающихся личностей. Интересен в этом аспекте и публикуемый сборник с работами В. А. Стеклова, в особенности, если рядом с ним мы упомянем вышедший недавно сборник работ А. М. Ляпунова по механике из его неопубликованного рукописного наследия [21].

Как известно, многие работы В.А. Стеклова и А. М. Ляпунова бывали тесно связаны и часто дополняли друг друга. Сюда относятся их результаты из гидродинамики, исследования задачи Дирихле и метода Неймана, связанные с ними результаты о свойствах потенциалов и т. д. Однако в их изложении, в творческой манере читаются принципиальные различия в характерах и устремлениях двух ученых.

А. М. Ляпунов по своей натуре был склонен к решению конкретных, сложнейших проблем. В этом было предназначение его гения. K точным решениям поставленных задач он двигался с неимоверной энергией и поистине титаническим терпением, преодолевая огромнейшие трудности. «Что особенно поражало у А. М. - это исключительная способность сосредоточивать свою мысль для достижения одной, главной цели. Даже общая теория устойчивости, глубоко разработанная им, была для него вспомогательной теорией, с помощью которой исследовалась устойчивость все тех же форм вращающейся жидкости $<\ldots>$. Получалось впечатление, что эта способность к сосредоточенности и углублению - какоето чисто структурное, физиологическое свойство природы самого организма». ${ }^{4}$ Исследование фигур равновесия вращающихся масс жидкости составляло, как известно, главную его задачу. Эту тему молодой Ляпунов получил от П. Л. Чебышева для своей магистерской диссертации и обращался к ней на протяжении всей своей жизни; в последние пятнадцать лет эти вопросы поглотили его совершенно, отдалив от других, современных ему, проблем математики и живого общения с ученым миром.

В. А. Стеклов в своей речи, посвященной этим трудам А. М Ляпунова и произнесенной на математическом конгрессе в Торонто (1924 г.), высказался так: «Исследования Ляпунова носят чисто аналитический характер; большинство страниц его рукописи содержат только очень сложные математические формулы, и его труд зачастую напоминает «песню без слов». $<\ldots>$ По-видимому, для этого выдающегося аналитика математический язык

${ }^{4}$ Из воспоминаний А. Д. Билимовича (А. Д. Билимович. А. М. Ляпунов в Одессе // Publication de l'institut mathématique (Beograd. Acad. Serbe des Sciences), T. IX, 1956, pp. 1-7).

НЕЛИНЕЙНАЯ ДИНАМИКА 2011. Т. 7. №2. С. 389-403 
был ясен сам по себе; и этого языка ему было достаточно для проникновения в «природу вещей» (in naturam rerum). ${ }^{5}$

У самого же В. А. Стеклова во всем его творчестве, куда входят также научно-популярные, философские, художественные произведения, обнаруживаются широкие, разносторонние интересы и живой, восприимчивый характер. В его научной работе видно влечение к генерированию идей, созиданию нового. Труды В. А. Стеклова, редко ограничиваясь одними вычислениями, попутно содержат интересные наблюдения, постановки новых нетривиальных задач.

С другой стороны, его активной натуре, видимо, не хватало спокойствия, необходимого для гармоничного завершения предпринятого исследования и строгого, выдержанного изложения. Так, например, открыв новый случай интегрируемости уравнений Кирхгофа главный результат диссертации [23], - В. А. Стеклов, к сожалению, не заметил взаимный интегрируемый случай (он был обнаружен и опубликован А. М. Ляпуновым [20]). Без сомнения, неспешная систематическая проработка намеченных проблем и более тщательная перепроверка выводов позволили бы ему гораздо полнее реализовать свои действительно замечательные, далеко идущие замыслы. Н. М. Гюнтер, ученик В. А. Стеклова, писал: «Его творческая мысль работала непрерывно, и возникавшие у него новые идеи претворялись им в дело немедленно. $<\ldots>$ Эта спешность бывала причиной недочетов, незаметных, когда видишь целое; но она же двигала дело - так, как новые мысли живут и рождают другие, только выпущенные в мир». ${ }^{6}$

И в самом деле, рассматриваемые здесь работы не отличаются отделанным изложением, их нельзя назвать безупречными. Однако важно отметить, что, действительно, при недостаточной строгости доказательств большинство выводов В. А. Стеклова в целом оказывается верно - во многом благодаря интуиции, общему пониманию вещей. Эти же качества подвели В. А. Стеклова к независимому рассмотрению действительно фундаментальных задач динамики. В постановках и решениях некоторых из них ему принадлежит приоритет. Многие его исследования соприкасаются с работами самых выдающихся его современников, начиная с А. Пуанкаре.

В классической механике, помимо значительных результатов по гидродинамике и движению твердого тела в жидкости, В.А. Стеклову принадлежат блестящие открытия в области динамики твердого тела, вошедшие в ее классический, «золотой» фонд (решение Бобылева-Стеклова, решение Стеклова уравнений Эйлера-Пуассона и др.).

Остановимся теперь подробнее на работах [1-8], для начала их перечислив в почти хронологическом порядке:

[1] (1902 г.): «Mémoire sur le mouvement d'un corps solide dans un liquide indéfini» («Meмуар о движении твердого тела в безграничной жидкости»);

[2] (1908 г.): «Sur la théorie des tourbillons» («O теории вихрей»);

\footnotetext{
${ }^{5}$ Stekloff $W$. Les recherches posthumes de Liapounoff sur les figures d'équilibre d'un liquide hétérogène en rotation // Proceedings of the International Mathematical Congress, Held in Toronto, August 11--16, 1924. Edited by J. C. Fields, Toronto: The University of Toronto Press, 1928, vol. 2, pp. 23-30. Ha pyc. яз.: Стеклов В.А. Посмертные труды Ляпунова о фигурах равновесия неоднородной вращающейся жидкости // Академик Ляпунов. Собрание сочинений: Т. 5, М.: Изд-во Акад. наук СССР, 1965. C. $379-384$.

${ }^{6}$ Н. М. Гюнтер. Труды В. А. Стеклова по математической физике // Усп. мат. наук, т. 1, вып. 3$4(13-14), 1946$, c. $23-43$.
} 
[3] (1905 г.): «Sur le problème du mouvement d'un ellipsoïde fluide homogène dont toutes les parties s'attirent suivant la loi de Newton» («K задаче о движении жидкого однородного эллипсоида, все части которого притягиваются друг к другу по закону Ньютона»);

[4] (1905 г.) и [5] (1906 г.): «Sur le mouvement non stationnaire d'un ellipsoïde fluide de révolution qui ne change pas sa figure pendant le mouvement» («О нестационарном движении жидкого эллипсоида вращения, который не изменяет конфигурации во время движения»);

[6] (1908 г.) и [7] (1909 г.): «Problème du mouvement d'une masse fluide incompressible de la forme ellipsoïdale les parties s'attirent suivant la loi de Newton» («Задача о движении несжимаемой жидкой массы, имеющей форму эллипсоида, частицы которой притягиваются друг к другу по закону Ньютона»).

[8] (1909 г.): «Sur le movement d'un corps solide ayant une cavité de forme ellipsoïdale remplie par un liquide incompressible et sur les variations des latitudes» («О движении твердого тела, имеющего полость эллипсоидальной формы, заполненную несжимаемой жидкостью, и об изменении широт»).

Статьи опубликованы В. А. Стекловым в журналах Comptes Rendus, Annales scientifiques de l'É.N.S и Annales de la faculté des sciences de Toulouse. Последний журнал был известен печатанием обширных мемуаров. Там же, кстати, были опубликованы переводы магистерской и докторской диссертаций А. М. Ляпунова. С другой стороны, тулузские «Анналы», по-видимому, использовались для быстрых публикаций, являясь своего рода «журналом препринтов».

Статья [1]. Работа 1902 года под названием «Мемуар о движении твердого тела в безграничной жидкости». По своему содержанию она является непосредственным продолжением и развитием магистерской диссертации [23], центральным результатом которой является нахождение В.А. Стекловым нового случая интегрируемости уравнений Кирхгофа движения твердого тела в идеальной жидкости.

В данном мемуаре В.А.Стеклов переходит к более сложной задаче и рассматривает движение неодносвязного твердого тела, в котором имеются отверстия и полости, наполненные идеальной несжимаемой жидкостью, совершающей потенциальные движения. В первой главе он выполняет расчет действующих в системе реакций, сил, давлений, пользуясь гидромеханическими соображениями. Отметим здесь, что ранее общий вывод уравнений для этой задачи был получен Томсоном (в его с Тэтом знаменитом трактате [41]) и Ламбом [35] в рамках обычного лагранжевого формализма с использованием обобщенных координат. В. А. Стеклов выводит общие уравнения движения системы в квазискоростях (т.е. в эйлеровских компонентах связанных с телом импульсивного момента и импульсивной силы). Этот способ позволил ему получить уравнения в наиболее явном и удобном для исследования виде. В той же самой форме эти уравнения имеются в работе Брайана [30], вышедшей, как и [23], в 1893 году, но, очевидно, прошедшей для Стеклова незамеченной. С выводом этих уравнений в современном виде можно ознакомиться, например, по книге [11]. Как известно, в случае неодносвязности твердого тела в гамильтониан уравнений Кирхгофа войдут слагаемые, линейные по компонентам импульсивной силы и импульсивного момента.

Во второй главе В. А. Стеклов пытается развить для уравнений Кирхгофа метод Пуанкаре нахождения общего решения всей системы в рядах по малому параметру; в качестве исходной невозмущенной системы он использует интегрируемый случай Эйлера-Пуансо. Этот же метод, как отмечает В. А. Стеклов, был применен Коббом [34] для нахождения периодических решений уравнений Эйлера-Пуассона задачи о вращении твердого тела вокруг

НЕЛИНЕЙНАЯ ДИНАМИКА 2011. Т. 7. №2. С. 389-403 
неподвижной точки. Нужно отметить, что, с современной точки зрения, В. А. Стеклов лишь доказал возможность продолжения по параметру (получения в форме рядов) перманентных вращений, порожденных (интегрированной) задачей Эйлера-Пуансо. Его прочие утверждения относительно продолжения остальных периодических решений невозмущенной задачи Эйлера-Пуансо не вполне корректны. Дело в том, что эти решения, действительно, являются вырожденными (в случае невозмущенной задачи) и при возмущении они, вообще говоря, разрушаются. В.А.Стеклов приводит ряд детерминантов, которые он полагает заведомо отличными от нуля, однако на самом деле они являются тождественно равными нулю.

Корректное решение этой задачи получается в рамках гамильтонова формализма $[9$, 10]. Следует произвести редукцию уравнений движения на общий уровень констант площадей геометрического интеграла. Тогда открывается и дальнейший путь: применить метод малого параметра Пуанкаре, относящийся к решениям гамильтоновых систем (а не к общим системам дифференциальных уравнений). Корректность получаемого в результате решения состоит в том, что мы имеем невырожденные невозмущенные решения (перманентные вращения Эйлера-Пуансо), которые можно продолжать по методу Стеклова, а остальные торы, вообще говоря, разрушаются, но при их разрушении возникают пары невырожденных резонансных торов, характеристический показатель которых также разлагается в ряд по степеням малого параметра, согласно теории Пуанкаре. Последнее как раз относится к рассматриваемым у В. А. Стеклова решениям заданного периода $T$.

Как мы видим, эти методы Пуанкаре довольно медленно пробивали себе дорогу, вызывая трудности даже у ведущих математиков. Их эффективная разработка была предпринята гораздо позже, в частности, в работах В. В. Козлова [15]. Недостатки анализа $[1,34]$ связаны и с тем, что авторам не хватило понимания гамильтоновой структуры уравнений Кирхгофа, хотя необходимые для того предпосылки, в принципе, уже были у обоих. Так, В.А. Стеклов обнаружил аналогию (аналогия Стеклова), которая сводит уравнения Кирхгофа к движению твердого тела вокруг неподвижной точки [40]. Г. Кобб же использовал канонические координаты для описания движения твердого тела (хотя в обобщенном виде это можно найти уже и у Якоби).

Статья [2]. Этот мемуар 1908 года под названием «О теории вихрей» посвящен исследованию общей задачи о вихревом движении жидкости, заполняющей некий произвольным образом движущийся сосуд. Это известная в гидродинамике задача; в различных постановках она изучается во многих работах (см., например, [19]). В. А. Стеклов в своем подходе опирается на разработанные им общие методы решения краевых задач математической физики [25]: его решение основано на сведении задачи о движении жидкости к решению задачи Неймана. (В своих сочинениях задачу Неймана он часто называет основной задачей гидродинамики.)

Он ставит задачу следующим образом. Пусть известны вихри $\omega_{1}, \omega_{2}, \omega_{3}$ заполняющей сосуд жидкости, т. е. задано вихревое распределение (поле вихрей) в каждой точке жидкости. Вихри являются функциями времени $t$ и координат точки $x, y, z$, т. е. зависят только от заданного закона движения сосуда. Требуется определить отсюда скорости точек $u, v, w$ жидкости, т.е. само течение. Решение такой задачи должно удовлетворять, помимо заданных начальных условий, некоторому условию на поверхности сосуда (т. е. граничному условию).

Сперва В.А. Стеклов рассматривает эту задачу в общем виде для случая, когда рассматриваемая жидкость ограничена некой замкнутой поверхностью $S$. Граничное условие выражается в равенстве нормальных составляющих скорости любой точки жидкости, примыкающей к поверхности сосуда, и скорости соответствующей точки этой поверхности. Последняя есть величина известная, поскольку закон движения сосуда задан наперед. 
Выражения для искомых скоростей жидкости получаются интегрированием дифференциальных уравнений, определяющих вихрь как функцию времени и координат точки, при выполнении заданного граничного условия.

В.А.Стеклов показывает, что решение задачи сводится к решению задачи Неймана для замкнутых поверхностей, т. е. к определению некоторой функции $P$, удовлетворяющей дифференциальным уравнениям вихрей и граничным условиям на $S$.

В результате он приходит к естественному выводу, что движение жидкости полностью определяется, если известно распределение вихрей во всех точках жидкости. На этом и основана главная идея предложенного в статье общего метода решения задачи. Этот метод автор называет «теорией вихрей» (отсюда происходит и название самой статьи).

В следующих параграфах В. А. Стеклов разрабатывает эту теорию на нескольких показательных примерах. Рассматривает различные частные случаи движения жидкости в замкнутом сосуде, каждый раз задавая соответствующее распределение вихрей.

В первом примере подробно рассматривается случай, когда сосуд представляет собой ограниченный (компактный) цилиндр вращения. Вихревые линии для этой задачи задаются в виде окружностей, лежащих в плоскостях, перпендикулярных оси вращения цилиндра. Устанавливается, что при заданных условиях движение жидкости возможно лишь в том случае, когда цилиндр осуществляет «геликоидальное», т. е. винтовое, движение. Сводя теперь решение к задаче Неймана, В. А. Стеклов получает искомые выражения для скоростей точек жидкости.

Показано, что аналогичным методом решается задача и в случае бесконечной жидкости. Граничное условие в этой постановке заменяется другим, при котором скорости жидкости на бесконечности обращаются в нуль (т. е. учитывается влияние ньютоновского потенциала). В. А. Стеклов дает решение этой задачи в предположении, что вихревые линии представляют собой окружности, лежащие в плоскости, перпендикулярной оси цилиндра, и с центром на этой оси.

Второй пример связан с движением жидкости в сосуде, ограниченном замкнутой произвольной поверхностью, в предположении, что вихревые линии являются параллельными прямыми с одинаковой интенсивностью. В частности, рассматривается случай, когда поверхность сосуда представляет собой трехосный эллипсоид, а частицы жидкости притягиваются друг к другу по закону Ньютона.

После этих предварительных выводов и получения основных формул В.А.Стеклов применяет свой общий метод к следующим двум классическим задачам.

Первая задача связана с нахождением уравнений движения твердого тела, имеющего эллипсоидальную полость, жидкость внутри которой совершает однородное вихревое движение (т. е. вихревые линии всегда остаются параллельными прямыми одинаковой интенсивности).

Примечательно, что В.А.Стеклов фактически выводит здесь те же уравнения, что двумя годами позже получил А. Пуанкаре [38]. Однако в отличие от Стеклова, опирающегося в своем выводе на общие гидродинамические соображения, Пуанкаре использует развитую им и тогда только входившую в обиход математиков теорию непрерывных групп преобразований (называемую теперь теорией групп и алгебр Ли). В современной литератуpe (например, [11]) эти уравнения обычно называют уравнениями Пуанкаре-Жуковского. Н.Е. Жуковский, как известно, указал их частный случай, интегрируемый при наличии осевой симметрии [14]. В наиболее общем виде они впервые получены именно В. А. Стекловым. Достойно сожаления, что его исследование осталось практически незамеченным. Конечно, в название этих уравнений следовало бы добавлять и имя Стеклова.

НЕЛИНЕЙНАЯ ДИНАМИКА 2011. Т. 7. №2. С. 389-403 
В статье [8] В. А. Стеклов указывает некоторые случаи интегрируемости для этой задачи. Кроме того, он использует полученные решения для обоснования явления прецессии земной оси. Этот мемуар, тесно связанный с вопросами динамики и физики планет, мы подробнее рассмотрим в дальнейшем.

Вторая задача относится к теории фигур равновесия. В. А. Стеклов выводит известные гидродинамические уравнения Римана, связанные с сохранением эллипсоидальной формы вращающейся жидкости. K этим уравнениям он добавляет еще уравнения поступательного движения тела, по-видимому, считая, что тем самым обобщает постановку Римана. Однако этот параметр является несущественным вследствие галилеевой инвариантности и может быть исключен простыми заменами координат.

Как известно, данная задача была поставлена и решена в лагранжевой форме Л. Дирихле [32, 33]. Б. Риман в своей знаменитой статье 1861 года [39] записал уравнения движения в новой, замкнутой форме. Подробный обзор работ по динамике жидких эллипсоидов и переводы основных классических статей по этой проблематике читатель найдет в вышедшем недавно сборнике «Динамика жидких и газовых эллипсоидов» [13].

В заключение В.А. Стеклов решает изложенным методом еще одну интересную задачу, связанную с течениями типа Бельтрами - движениями, для которых линии течения совпадают с вихревыми линиями. Он определяет некоторые частные течения (их, вообще говоря, немного), пользуясь для их нахождения некой итерационной процедурой и методом Робина. В наше время такие потоки, ныне называемые АВС-течениями ${ }^{7}$, приобрели особый интерес в их связи с изучением свойств глубокого перемешивания и гидродинамического хаоса, начиная с пионерских работ Эно и Арнольда.

Статьи [3-5]. Три краткие заметки годов 1905-1906 из Comptes rendus, в которых В.А.Стеклов, как ему показалось, нашел новые решения задачи Римана-Дирихле о движении жидкого эллипсоида. Мы не будем отдельно останавливаться на обсуждении этих заметок. Они являются, по сути, предварительными сообщениями результатов, изложенных затем в двух обширных статьях $[6,7]$, рассмотренных ниже.

Статьи [6, 7]. Обе работы посвящены вопросам, связанным с задачей ДирихлеРимана о движении жидкого эллипсоида. Они были опубликованы в 1908-1909 годах в журнале Annales scientifiques de l'É.N.S под общим заглавием «Задача о движении несжимаемой жидкой массы, имеющей форму эллипсоида, частицы которой притягиваются друг к другу по закону Ньютона».

Первая статья [6] вышла в том же году, что и рассмотренная выше работа по теории вихрей [2]. Опираясь на свой вихревой метод, В.А.Стеклов выводит уравнения Римана и отмечает любопытную аналогию этой задачи с задачей о движении твердого тела с полостями, заполненными жидкостью. По-видимому, В. А. Стеклов был первым и единственным, кто стал рассматривать две эти задачи совместно. Во всяком случае, А. Пуанкаре в своем выводе [38] никак не упоминает задачу Римана, хотя она уже была разрешена также в гамильтоновой форме со скобкой Пуассона на алгебре Ли.

Однако основной целью работы [6], очевидно, является пересмотр теоремы Римана. Стеклову показалось, что он нашел опровержение общего утверждения Римана о том, что «с постоянством формы неизбежно связано постоянство движения», т. е. всякий раз, когда жидкая масса образует одно и то же тело, относительное движение всех частиц этого тела остается неизменным [39]. Решая задачу отыскания всех возможных движений жидкости

${ }^{7}$ По английской аббревиатуре, составленной именами Арнольда, Бельтрами и Чилдресса. 
в случае эллипсоида вращения, Стеклов, как ему кажется, находит такие нетривиальные частные случаи, когда неизменной форме свободной поверхности эллипсоида соответствуют нестационарные внутренние течения. В своем анализе он применяет метод неопределенных коэффициентов. Считая свои выводы важными, В.А.Стеклов предварительно в сжатой форме опубликовал их в виде трех заметок [3-5].

Впрочем, в самом начале следующей статьи [7], продолжения этого мемуара, В. А. Стеклову приходится признать свои выводы касательно теоремы Римана неверными. Он приводит замечания из письма С. А. Чаплыгина, заключающиеся в том, что если указанные Стекловым движения рассматривать, перейдя во вращающуюся систему координат, то они сразу сводятся к стационарному типу и, таким образом, утверждение Римана верно и в самом общем случае. Сам С. А. Чаплыгин по этим вопросам ничего не опубликовал, хотя его рукописное наследие свидетельствует о том, что он много занимался задачами динамики самогравитирующей жидкости.

Разобравшись таким образом со случаем эллипсоида вращения, В. А. Стеклов переходит к изучению возможных движений в случае несимметричного эллипсоида. Этим исследованием он, по сути, доказывает теорему Римана о постоянстве формы и движения в общей форме.

Отметим, что, хотя в своей работе [39] Б. Риман приводит лишь формулировку данной теоремы, представляется маловероятным, чтобы он публиковал ее, не владея строгим доказательством. Возможно, он позволил себе не приводить его, отнеся к деталям своего выдающегося исследования. Подобных опущенных «деталей», видимо, совершенно очевидных для риманового гения, но представляющих большие, отдельные задачи для других исследователей, в [39], действительно, немало. По возможностям дальнейших важных обобщений работа Римана и сегодня является уникальной.

Далее исследуется интегрируемость полученных уравнений движения. Как и большинство механиков того времени, не владеющих еще понятием гамильтоновости уравнений движения, В.А. Стеклов использует для определения интегрируемости стандартный метод последнего множителя Якоби (теорему Эйлера-Якоби).

Этот анализ представляет теперь лишь исторический интерес. Корректный подход заключается в определении интегрируемости системы по Лиувиллю, для чего следует воспользоваться гамильтоновой формой уравнений Римана. Эту задачу еще в 1881 году фактически разрешил итальянский математик и тополог Бетти в малоизвестной работе [28]. Бетти представил уравнения движения на скобках Пуассона и, используя коммутацию на so(4), выполнил редукцию системы на несколько степеней свободы, таким образом сведя задачу интегрирования уравнений Римана к задаче нахождения полного интеграла уравнения Гамильтона-Якоби.

Статья [8]. Обширный мемуар 1909 года «О движении твердого тела, имеющего полость эллипсоидальной формы, заполненную несжимаемой жидкостью, и об изменении широт».

Здесь В.А. Стеклов наиболее полным, систематическим образом исследует уравнения движения для своей (поставленной в [2]) задачи о движении твердого тела с эллипсоидальной полостью, жидкость внутри которой совершает однородное вихревое движение. В отличие от общей постановки [2], здесь он выписывает уравнения в предположении, что центр эллипсоида неподвижен, жидкость совершает движение Дирихле.

Для интегрирования соответствующих уравнений он применяет метод последовательных приближений в предположении, что масса жидкости достаточно мала по отношению к массе тела. Далее рассматриваются некоторые частные решения.

НЕЛИНЕЙНАЯ ДИНАМИКА 2011. Т. 7. №2. С. 389-403 
Основным результатом этой части работы является указание нового интегрируемого семейства для данной задачи. Как выяснилось намного позднее, этот новый случай Стеклова оказался тесно связан с известным семейством Стеклова-Ляпунова в теории уравнений Кирхгофа - двумя взаимными интегрируемыми случаями, первый из который был получен В.А.Стекловым в [23], а второй - А. М.Ляпуновым в [20]. В современных работах показано, что случай Стеклова сводится к случаю Стеклова-Ляпунова путем ретракции, и установлен их линейный изоморфизм (см. книгу [11]). Недавно наиболее наглядный способ явного интегрирования этого случая был предложен А. В. Цыгановым [42].

Далее В. А. Стеклов пытается получить обширные периодические семейства своих уравнений движения, применяя к ним метод малого параметра Пуанкаре. Поскольку он не знает гамильтоновой структуры этих уравнений (она представляет собой алгебру so(4)), его анализ в этой части работы страдает теми же недостатками, что мы уже отмечали выше при обсуждении второй главы мемуара [1] (где он аналогичным образом подходит к уравнениям Кирхгофа). Действительно, большинство долгопериодических решений, лежащих на резонансных торах, являются вырожденными. В.А.Стеклов строго показывает только невырожденность и возможность продолжения по параметру перманентных вращений задачи Эйлера.

Отметим, что в общем виде эта проблема не решена до сих пор: остается вопрос нахождения семейств периодических решений этой задачи, а значит, остается неисследованным и вопрос, связанный с доказательством неинтегрируемости. Ведь, как известно, по теоремам Пуанкаре, существование большого числа невырожденных периодических решений несовместимо с интегрируемостью задачи.

Полученные выше результаты, как это видно из названия статьи, В. А. Стеклов применяет к задаче из геодинамики, связанной с вариациями земных широт вследствие колебаний оси вращения Земли.

Феномен движения земных полюсов был обнаружен во второй половине XIX века путем многолетних наблюдений; его открытие связывают с работами американского астронома С. К. Чандлера. Это открытие послужило мощным стимулом для развития более строгих теорий физики и динамики планет. Как известно, основополагающими в развитии этих исследований явились идеи лорда Кельвина. Он первым, в связи со своей теорией земных приливов и отливов, предложил модель земного шара как твердотельной недеформируемой эллипсоидальной мантии, заключающей в себе эллипсоидальное ядро из однородной идеальной жидкости. Используя эту модель в изучении явлений прецессии и нутации земной оси, Кельвин пришел к выводу, что вся система (и мантия, и внутренняя жидкость) должна вращаться как одно сплошное твердое тело тело, т. е. имеет место так называемый эффект гиростатической жесткости. Этот результат опрокинул бытовавшие тогда представления геологов о Земле как о достаточно тонкой коре с заполняющим ее жидким ядром. С теорией лорда Кельвина читатель теперь может ознакомиться по недавно вышедшему русскому изданию знаменитого трактата Томсона (лорда Кельвина) и Тэта [41]. Подробное изложение наиболее значительных результатов по теории внутреннего строения Земли, начиная с исследований Кельвина, Пуанкаре, Лява и др., можно найти, например, в классических книгах $[36,44]$.

В. А. Стеклов в своем исследовании развивает модель Земли, объясняющую отдельные особенности движения земных полюсов влиянием, которое может оказывать на движение эллипсоидальной твердой оболочки движение внутренней жидкости. Используя свою теорию периодических решений, он делает ряд выводов, согласующихся с астрономическими наблюдениями; в частности, получает уравнения для периода чандлеровской прецессии. 
Кроме того, найденные решения позволяют ему сделать численные выводы относительно толщины твердой земной оболочки. Отметим, что В. А. Стеклов получает здесь те же уравнения для периода Чандлера, что ранее Вито Вольтерра [43] (1899 г.). Вольтерра в своем решении, однако, исходил из других соображений: он использовал другую модель, сведя задачу о движении тела, содержащего полость, к изучению движения гиростата.

Более строгое и совершенное теоретическое обоснование эффектов прецессии и нутации было дано Пуанкаре в его знаменитой работе «О прецессии деформируемых тел» [38]. Пуанкаре исследовал резонансную природу колебаний Земли и открыл явление двойного резонанса, при котором система перестает вести себя как сплошное твердое тело, т.е. кельвиновская гиростатическая жесткость имеет место лишь как частный случай общего явления, соответствующий простому резонансу.

Отметим, что некоторые закономерности движения земных полюсов не прояснены до сих пор, хотя сегодня существуют и более детальные модели Земли, учитывающие влияние многих дополнительных параметров. Современная модель предполагает наличие в самом центре Земли еще одного, твердого, ядра. Однако, в силу своей сложности, эти модельные задачи пока не дают полного и строго решения. Простая классическая модель, рассматриваемая Стекловым и Пуанкаре, является одной из наиболее естественных - ее можно исследовать современными качественными методами теории динамических систем.

В дополнение к упомянутым основным результатам, в этой работе В. А. Стеклов приводит в явном виде особые решения для различных типов поверхностей полости. Они представляют собой некие стационарные решения, подобные тем перманентным вращениям, которые он находил ранее в динамике твердого тела.

Кроме того, им анализируется влияние вязкости внутренней жидкости на характер движения системы. Показано, что при добавлении линейной стоксовой вязкости жидкость также будет совершать движения Дирихле, но в этом случае она будет прилипать к стенкам эллипсоидальной полости. Вообще говоря, изучение динамики тел с полостями, содержащими жидкость, при наличии вязкости является весьма сложной задачей. Подробную библиографию по этой проблематике читатель найдет, например, в книге [22]. Из более современных исследований упомянем, в частности, работы С. Н. Судакова ([26] и др.).

Задача Стеклова о падении тяжелого твердого тела в идеальной жидкости. В дополнение к вышеописанным работам, отметим здесь еще одно исследование В. А. Стеклова по динамике твердого тела в жидкости. Оно содержится в его диссертации [23, глава V], но, хоть и опубликованное на русском языке, оказалось незамеченным и потому заслуживает упоминания хотя бы в качестве примечательного исторического факта.

В этом исследовании В.А. Стеклов впервые рассмотрел задачу о движении тяжелого твердого тела в безграничном объеме идеальной жидкости. (В предшествующих публикациях рассматривалось движение тела по инерции, без учета силы тяжести.) Кроме того, В.А. Стеклов был первым, кто, получив для этой задачи уравнения движения, попытался исследовать их качественно. Он выделил случай падения тела с плоскостью симметрии, т.е. случай плоско-параллельного движения, получил для него уравнение движение $(\ddot{x}=$ $\left.=t^{2} \sin \varphi \cos \varphi\right)$ и поставил задачу асимптотического описания поведения его решений. Он, в частности, отметил простейшие их свойства, сформулировал несколько теорем, устанавливающих асимптотическое поведение тела при $t \rightarrow \infty$.

Выводы В.А. Стеклова большей частью правильны, но сам путь их доказательства, к сожалению, недостаточно строг и содержит много упущений в вычислениях. (К этим 
вычислениям, кстати, и относится основная критика диссертации А. М. Ляпуновым.) Часть отмеченных погрешностей В. А. Стеклов исправил в заметке [24] (дополнение к [23]). В частности, он включил в нее вывод, что при падении тела амплитуда его колебаний относительно горизонтальной оси убывает, а частота колебаний растет. Интересно отметить, что А. М. Ляпунов, указав все неточности анализа [23], тем не менее, не взялся сам получить строгое решение этой задачи, хотя (с точки зрения развития разработанных им методов теории устойчивости) такое исследование было бы весьма интересно.

То же уравнение плоско-параллельного движения тела в идеальной жидкости было получено С. А. Чаплыгиным в его студенческом сочинении 1890 года [27]. От публикации этого результата С. А. Чаплыгин воздержался, возможно, потому, что ему не удалось проинтегрировать это уравнение в явном виде. (Как известно, явное разрешение задач в квадpaтypax С.А. Чаплыгин считал основной задачей динамики, в нем и состоит большинство его выдающихся результатов.) Эта рукопись попала в печать лишь через сорок лет, с выходом первого, прижизненного, собрания его трудов. Тем не менее, в литературе задачу о падении тяжелого твердого тела в идеальной жидкости связывают, как правило, только с именем С. А. Чаплыгина.

Полный качественный анализ задачи Стеклова об асимптотическом описании поведения решений уравнения и их устойчивости был выполнен В. В. Козловым [16], который показал, что при почти всех начальных условиях тело стремится к равноускоренному падению широкой стороной перпендикулярно полю тяжести и колеблется вокруг горизонтальной оси с возрастающей частотой порядка $t$ и уменьшающейся амплитудой порядка $1 / \sqrt{t}$. Более современный анализ этой и родственных ей задач см. [12]; задача о падении тяжелого твердого тела в идеальной жидкости при ненулевой циркуляции разобрана в работе [29].

Авторы данного очерка располагают оригинальными текстами большинства статей, цитируемых в списке литературы, а также в самих работах В. А. Стеклова, и готовы выслать копии заинтересованному читателю по запросу. Ряд классических работ находится в полнотекстовом свободном доступе в Интернете (см. архивы журналов и собраний сочинений на порталах Numdam, Gallica, Gallica-Math, Ebook and Texts Archive и др.).

\section{Список литературы}

[1] Stekloff V.A. Mémoire sur le mouvement d'un corps solide dans un liquide indéfini // Ann. Fac. Sci. Toulouse Math. (2), t. 4, 1902, pp.171-219. (Статья I.)

[2] Stekloff V.A. Sur la théorie des tourbillons // Ann. Fac. Sci. Toulouse Math. (2), 1908, vol. 10, pp. 271-334. (Статья II.)

[3] Stekloff V. A. Sur le problème du mouvement d'un ellipsoïde fluide homogène dont toutes les parties s'attirent suivant la loi de Newton // C. R. Acad. Sci. Paris, 1905, vol. 141, pp.999-1001. (Статья III.)

[4] Stekloff V.A. Sur le mouvement non stationnaire d'un ellipsö̈de fluide de révolution qui ne change pas sa figure pendant le mouvement // C. R. Acad. Sci. Paris, 1905, vol. 141, pp. 1215-1217. (Статья IV.)

[5] Stekloff V.A. Sur le mouvement non stationnaire d'un ellipsoïde fluide de révolution qui ne change pas sa figure pendant le mouvement // C. R. Acad. Sci. Paris, 1906, vol. 142, pp. 77-79. (Статья V.)

[6] Stekloff V.A. Problème du mouvement d'une masse fluide incompressible de la forme ellipsoïdale les parties s'attirent suivant la loi de Newton // Ann. Sci. École Norm. Sup. (3), 1908, vol. 25, pp. 469-528. (Статья VI.) 
[7] Stekloff V. A. Problème du mouvement d'une masse fluide incompressible de la forme ellipsoïdale 1 es parties s'attirent suivant la loi de Newton (Suite) // Ann. Sci. École Norm. Sup.(3), 1909, vol. 26, pp. 275-336. (Статья VII.)

[8] Stekloff V. A. Sur le movement d'un corps solide ayant une cavité de forme ellipsoïdale remplie par un liquide incompressible et sur les variations des latitudes // Ann. Fac. Sci. Toulouse Math. (3), 1909, vol. 1, pp. 145-256. (Статья VIII.)

[9] Баркин Ю.В., Борисов А.В. Неинтегрируемость уравнений Кирхгофа и родственных задач динамики твердого тела: Депонировано в ВИНИТИ, 5037-В89. М., 1989.

[10] Борисов А. В. Необходимые и достаточные условия интегрируемости уравнений Кирхгофа // Регулярная и хаотическая динамика, 1996, т. 1, № 2, с. 61-76.

[11] Борисов А. В., Мамаев И. С. Динамика твердого тела: Гамильтоновы методы, интегрируемость, хаос. М.-Ижевск: ИКИ, 2005. 576 с.

[12] Борисов А. В., Козлов В. В., Мамаев И. С. Асимптотическая устойчивость и родственные задачи динамики падающего тяжелого твердого тела // Нелинейная динамика, 2007, т. 3, № 3, с. 255296.

[13] Динамика жидких и газовых эллипсоидов / А. В. Борисов, И. С. Мамаев (ред.). М.-Ижевск: НИЦ «РХД», 2010. 364 с.

[14] Жуковский Н. Е. О движении твердого тела, имеющего полости, наполненные однородной капельной жидкостью: I, II, III // Собр. соч.: Т. 1. М.-Л.: ОГИЗ, 1949. С. 31-152. (1-е изд.: Журн. рус. физ.-хим. общества, 1885, т. 17, отд. 1, вып. 6, с. 81-113; вып. 7, с. 145-149; вып. 8, с. 231280.)

[15] Козлов В. В. Методы качественного анализа в динамике твердого тела. М.: Изд-во Моск. ун-та, 1980. 231 с. (2-е изд., дополненное: М.-Ижевск: НИЦ «РХД», 2000. 248 с.)

[16] Козлов В.В. О падении тяжелого твердого тела в идеальной жидкости // Изв. АН СССР, MTT, 1989, № 5, с. 10-17.

[17] Козлов В.В. Симметрии, топология и резонансы в гамильтоновой механике. Ижевск: Издво Удмуртского гос. ун-та, 1995. 429 с.

[18] Козлов В. В. Общая теория вихрей. М.-Ижевск: НИЦ «РХД», 1998. 238 с.

[19] Кочин Н.Е., Кибель И. А., Розе Н. В. Теоретическая гидромеханика: Т. 1. М.: ГИТТЛ, 1955. $560 \mathrm{c}$.

[20] Ляпунов А. М. Новый случай интегрируемости уравнений движения твердого тела в жидкости // Собр. соч.: Т. 1. М.: Изд-во Акад. наук, 1954. С. 320-324. (1-е изд.: Сообщ. Харьк. матем. общ-ва, сер. 2, 1893, т. 4, № 1-2, с. 81-85.)

[21] Ляпунов А. М. Работы по теоретической механике. Из рукописного наследия 1882-1894 годов. М.-Ижевск: НИЦ «РХД», 2010. 460 с.

[22] Моисеев Н. Н., Румянцев В. В. Динамика тела с полостями, содержащими жидкость. М.: Наука, 1965. $440 \mathrm{c}$.

[23] Стеклов В. А. О движении твердого тела в жидкости. Харьков, 1893. xvi +234 c.

[24] Стеклов В. А. Дополнение к сочинению «О движении твердого тела в жидкости» // Сообщ. Харьк. мат. общ-ва (2), 1894, т. 7, № 4, с. 161-162.

[25] Стеклов В. А. Общие методы решения основных задач математической физики. Харьков, 1901. $\mathrm{v}+291 \mathrm{c}$.

[26] Судаков С. Н. Модельная задача о движении вокруг центра масс абсолютно твердой эллипсоидальной оболочки с вязко-упругим заполнением // МТТ, 2003, вып. 33, с. 1-8.

[27] Чаплыгин С. А. О движении тяжелых тел в несжимаемой жидкости. (Студенческое сочинение, написанное в 1890 году. Впервые опубликовано в «Полном собрании сочинений С. А. Чаплыгина», т. 1, 1933, с. 312-336; см. также: Чаплыгин С. А. Собр. соч.: Т. 1: Теоретическая механика. Математика. М.-Л.: ГИТТЛ, 1948. 484 с.) 
[28] Betti E. Sopra i moti che conservano la figura ellissoidale a una massa fluida eterogenea // Annali di Matematica Pura ed Applicata, Serie II, 1881, vol. 10, pp. 173-187 [Бетти Э. О движениях, сохраняющих эллипсоидальную форму // Динамика жидких и газовых эллипсоидов / А. В. Борисов, И. С. Мамаев (ред.). М.-Ижевск: НИЦ «РХД», 2010. С. 134-149].

[29] Borisov A. V., Mamaev I. S. On the motion of a heavy rigid body in an ideal fluid with circulation // Chaos, 2006, vol. 16, no. 1, 013118 (7 pp.).

[30] Bryan G.H. A Hydrodynamical proof of the equations of motion of a perforated solid, with applications to the motion of a fine rigid framework in circulating liquid // Proceedings of the Physical Society of London, 1892, vol. 12, issue 1, pp. 186-204.

[31] Carathéodory C. Calculus of variations and partial differential equations of the first order: Part 1. Partial differential equations of the first order. San Francisco-London-Amsterdam: Holden-Day, Inc., 1965. $171 \mathrm{p}$.

[32] Dirichlet G.L. Untersuchungen über ein Problem der Hydrodynamik // Nachrichten von der Gesellschaft der Wissenschaften zu Göttingen (Mathematisch-Physikalische Klasse), 1857, No. 14, S. 203-207 (Dirichlet's Werke, Bd. 2, S. 28) [Дирихле Л. Исследование одной задачи гидродинамики // Динамика жидких и газовых эллипсоидов / А. В. Борисов, И. С. Мамаев. М.-Ижевск: НИЦ«РХД», 2010. С. 16-19].

[33] Dirichlet G.L. Untersuchungen über ein Problem der Hydrodynamik (Aus dessen Nachlass hergestellt von Herrn R. Dedekind zu Zürich) // J. Reine Angew. Math. (Crelle's Journal), 1861, Bd. 58, S. 181-216 [Дирихле Л. Исследование одной задачи гидродинамики // Динамика жидких и газовых эллипсоидов / А. В. Борисов, И. С. Мамаев. М.-Ижевск: НИЦ «РХД», 2010. С. 19-58].

[34] Kobb G. Sur le problème de la rotation d'un corps autour d'un point fixe // Bulletin de la S. M. F., 1895, vol. 23, pp. 210-215 [Кобб Г. Задача о вращении тела вокруг неподвижной точки // Система Клебша. Разделение переменных, явное интегрирование? / А. В. Борисов, А. В. Цыганов (ред.). М.-Ижевск: НИЦ «РХД», 2009. С. 159-165].

[35] Lamb H. Hydrodynamics. 6th ed. New York: Dover, 1945 [Ламб Г. Гидродинамика. М.-Л.: ОГИЗ, Гостехиздат, 1947. 928 с. Репринт: Том 1, 2. М.-Ижевск: НИЦ «РХД», 2003].

[36] Melchior P. Physique et dynamique planétaires: Vol. 1, 2. Bruxelles: Vander, 1971-1972. 247 p.; 311 p. [Мельхиор П. Физика и динамика планет: В 2-х тт. М.: Мир, 1975. 576 с.; 484 с.]

[37] Olver P. Applications of Lie groups to differential equations. New York: Springer, 1986. 497 р. [Олвер П. Приложения групп Ли к дифференциальным уравнениям. М.: Мир, 1989. 638 с.]

[38] Poincaré H. Sur la precession des corps deformables // Bull. Astr., 1910, vol. 27, pp. 321-356 [cм. Пуанкаре А. Последние работы. М.-Ижевск: НИЦ «РХД», 2001. С. 72-73].

[39] Riemann B. Ein Beitrag zu den Untersuchungen über die Bewegung eines flüssigen gleichartigen Ellipsoïdes // Abh. d. Königl. Gesell. der Wiss. zu Göttingen, 1861 [Риман Б. Сочинения. М.Л.: ГИТТЛ, 1948. С. 339-366; а также см.: Риман Б. О движении жидкого однородного эллипсоида // Динамика жидких и газовых эллипсоидов / А. В.Борисов, И. С. Мамаев (ред.). М.-Ижевск: НИЦ «РХД», 2010. С. 74-107].

[40] Stekloff V.A. Remarque sur un problème de Clebsch sur le mouvement d'un corps solide dans un liqiude indefini en sur le problème de M. de Brun // C. R. Acad. Sci. Paris, 1902, vol. 135, pp. 526-528 .

[41] Tait P. G. and Thomson W. Treatise on natural philosophy: Vol. 1, 2. Cambridge: Cambridge Univ. Press, 1867 [Томсон У. (лорд Кельвин), Тэт П. Г. Трактат по натуральной философии: В 2-х тт. М.-Ижевск: НИЦ «РХД», 2010. 572; 592 с.].

[42] Tsiganov A. V. On the Steklov-Lyapunov case of the rigid body motion // Regul. Chaotic Dyn., 2004, vol. 9, no. 2, pp. 77-91.

[43] Volterra V. Sur la théorie des variations des latitudes // Acta Math., 1899, vol. 22, pp. 201--358.

[44] Woolard E. W. Theory of the rotation of the Earth around its center of mass // Astronomical papers prepared for the use of the American ephemeris and nautical almanac, vol. 15, part 1. Washington, 
U.S. Govt. Print. Off., 1953 [i. е. 1954], 165 р. [Вулард Э. Теория вращения Земли вокруг центра масс. М.: Физматгиз, 1963. 143 с.]

\section{On V.A.Steklov's legacy in classical mechanics}

Alexey V. Borisov ${ }^{1}$, Larisa A. Gazizullina ${ }^{2}$, Ivan S. Mamaev ${ }^{3}$

Institute of Computer Science, Udmurt State University

1, Universitetskaya st., Izhevsk 426034, Russia

${ }^{1}$ borisov@rcd.ru, ${ }^{2}$ lag@rcd.ru, ${ }^{3}$ mamaev@rcd.ru

The paper is written for the edition of V. A. Steklov's collected French-language papers in mechanics.

Received January 17, 2011

Citation: Rus. J. Nonlin. Dyn., 2011, vol. 7, no. 2, pp. 389-403 (Russian) 